\title{
ROLE OF CRYSTAL-MELT INTERFACE AND SURFACE TENSION ON CZOCHRALSKI GROWTH OF SUBSTRATE MATERIALS WITH $\mathrm{K}_{2} \mathrm{NiF}_{4}$ STRUCTURE
}

\author{
M. Berkowski, J. Fink-Finowicki and M. Gutowski \\ Institute of Physics, Polish Academy of Sciences \\ Al. Lotników 32/46, 02-668 Warsaw, Poland
}

\begin{abstract}
Crystal-melt interface was investigated during Czochralski growth of $\mathrm{SrLaGaO}_{4}$ and $\mathrm{SrLaAlO}_{4}$ crystals on $\langle 001\rangle$ oriented seeds. Relations between the ratio of the core diameter, grown on (001) plane, and the crystal diameter, as a function of seed rotation speed were determined. It was confirmed that it is possible to control the core diameter during the crystal growth. A new module was introduced into the crystal growth controlling program. It enables to estimate the surface tension coefficient between crystal and melt at the beginning of crystallization. This value is then used to compute proper corrections for automatic weighting system.
\end{abstract}

PACS numbers: $81.10 .-\mathrm{h}, 81.10 . \mathrm{Fq}, 47.20 . \mathrm{Dr}, 68.10 . \mathrm{Cr}$

\section{Introduction}

Single crystals, obtained by the Czochralski method at low seed rotation rate and typical thermal configuration, keep convex crystal-melt interface during the growth. The central part of the crystal (core region - about $25 \%$ of the crystal diameter) has the composition slightly different from that in the outer part (facets region) which results in the creation of internal stresses.

In order to obtain good quality single crystals, the vertical temperature gradicnt above the melt should be minimized. The highest vertical temperature gradient exists just above and near the melt surface. Depending on the thermal configuration used, the mean value of the vertical temperature gradient in the region between melt surface and upper edge of a crucible falls in the range $30-45 \mathrm{~K} / \mathrm{mm}$ [1]. This high gradient can be reduced to about $8 \mathrm{~K} / \mathrm{mm}$ when the active afterheater and the special RF coil, consisting of two separated groups of windings, is applied [2]. The lower part of the coil heats the crucible whereas the upper part (one or two turns) heats the afterheater. Above the upper edge of the crucible the typical value of vertical temperature gradient is $1-3 \mathrm{~K} / \mathrm{mm}$. The value of temperature 
gradient in this region depends mostly on the afterheater length. It seems that the most effective way to minimize vertical temperature gradient just above and near the melt surface is application of a long, active afterheater placed directly on the crucible and covered with a ring disc.

\section{Crystal-melt interface}

It is known that the best quality of the crystal can be achieved when crystal growth takes place on a single F-type crystallographic plane. The most important F-type plane, revealing during the crystal growth of these materials, is (001). In the crystal grown on $\langle 001\rangle$ oriented seed the core region is formed by the central part grown on (001) plane, whereas the outside facets region is grown on four (103)-type planes. Therefore the crystal-melt interface was investigated in $\mathrm{SrLaGaO}_{4}$ crystals pulled on $\langle 001\rangle$ oriented seeds. Constant crystal/crucible diameter ratio and fixed thermal configuration were used during crystal growth processes. These studies allow to determine the relation between the ratio of the core diameter, grown on (001) plane, and the crystal diameter, as a function of seed rotation as shown in Fig. 1. The results obtained confirm that it is possible to control the core diameter during the crystal growth.

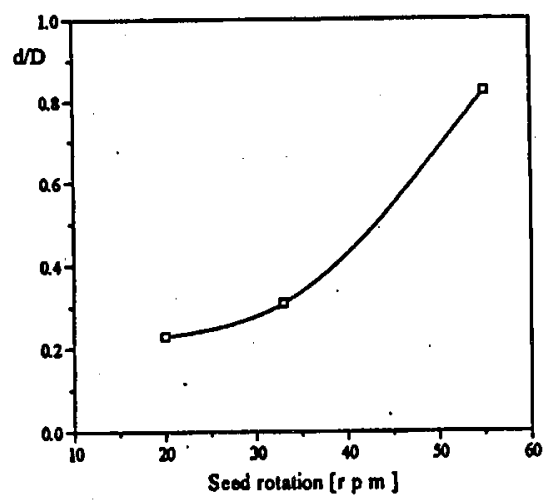

Fig. 1. The ratio of the core diameter $(d)$ to the crystal diameter $(D)$ as a function of seed rotation [rpm]. The $\mathrm{SrLaGaO}_{4}$ crystals are grown on $\langle 001\rangle$ oriented seed. Crucible diameter $50 \mathrm{~mm}$, equivalent crystal's diameter $21.5 \mathrm{~mm}$.

\section{Surface tension}

Finishing the crystallization process is always accompanied with a certain thermal shock. This is because one has to separate the crystal from a melt and relocate it at least several $\mathrm{mm}$ over the melt's surface. Basically, two different methods are used to end the crystallization. The easier one is to quickly remove the crystal from the melt. The main disadvantage of this method is an abrupt change of thermal flux flowing through and dissipated by the crystal. Another way is to increase gradually the melt's temperature in order to reduce the crystal's diameter and discontinuing the crystal-melt contact only after that. Application 
of this method may significantly reduce the sudden changes of thermal flux. There are, however, severe technical difficulties related to the lack of precise control over the crystal diameter during this phase of growth. The difficulties are connected with appearance of surface tension forces acting between the crystal and melt. The nature of these forces is complex and they are usually not taken into account by the automatic system controlling the crystal growth process. The error, made during evaluating of the mass of growing crystal and related to the forces of surface tension, is relatively large. It plays practically no role when the crystal diameter is constant (seed prolongation and cylindrical part) but manifests itself significantly when the crystal diameter is changing (conical parts - the beginning and end of crystal growth process). The change of weight readouts during disconnection of crystal from melt is shown in Fig. 2. The shape of curves presented there, as well as

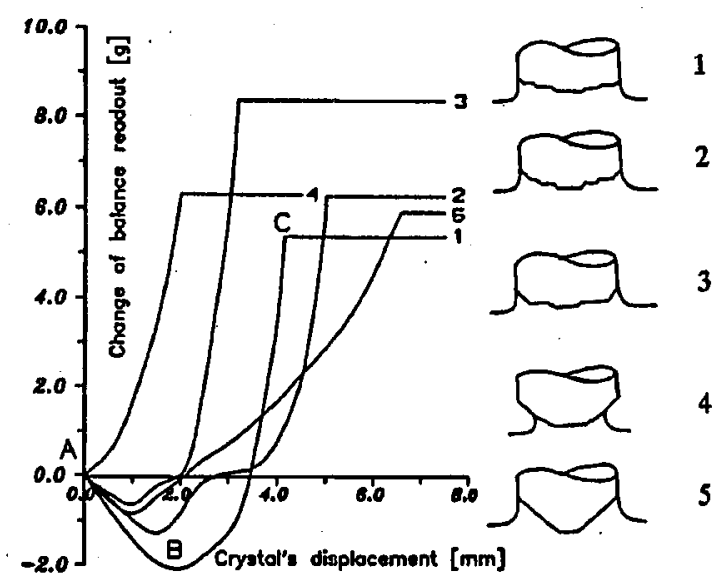

Fig. 2. Change of balance readout versus crystal displacement during disconnection of crystal from the melt. $A$ - beginning of disconnection of crystal from the melt, $A B$ - conservation of initial circumference wetted by melt regardless of increased height of elevated melt, $B-$ melt reaches wetting angle at the end of crystal, $B C-$ gradual decrease in wetted circumference of crystal, $C$ - disconnection.

the values of forces of surface tension, depend not only on crystal's circumference but also on the shape of crystal-melt interface. It is also influenced by the current phase of crystal growth, i.e. whether during disconnection the cylindrical or conical part of crystal was growing; see curves 1 and 4 . The total force of surface tension, monitored by balance, may be expressed by the formula

$$
F=\pi D \sigma \cos \theta
$$

where $\pi D$ - circumference of immersed part of crystal, $\sigma$ - coefficient of surface tension, $\Theta$ - angle between vertical direction and a line tangent to the melt's surface at the boundary with crystal (the balance measures only vertical components of all existing forces). From the above formula it is clear that the relevant factors are the crystal's diameter and the angle $\Theta$. Additionally, earlier described factors are evolving in time, thus making the precise calculations impossible. Therefore, 
one has to model this part of growth process rather than simply calculate it. More detailed discussion of this problem is presented elsewhere [3].

\section{Summary}

Transition from phase of seed prolongation to growth of conical part of crystal is connected with sudden increase in angle $\theta$, i.e. significant decrease in surface tension forces. This change is incorrectly interpreted by controlling program as a decrease in crystal's diameter. As a result, the growth process becomes difficult to control.

Inclusion of a new module, which enables the measurements of surface tension coefficient between crystal and melt at the beginning of crystallization, into the crystal growth controlling program permits to accurately evaluate changes of crystal's diameter/mass ratio and to avoid the oscillatory character of growth in this region.

Moreover, taking into account of the surface tension forces made also possible smooth gradual decrease in crystal diameter at the end of crystal growth process. This reduces significantly thermal shock when the contact between crystal and melt is disrupted.

\section{Acknowledgments}

This work was partially supported by the Commission of the European Communities under contract CIPA-CT93-0032.

\section{References}

[1] M. Sasaura, S. Miyazawa, J. Cryst. Growth 131, 413 (1993).

[2] R. Uecker, P. Raiche, V. Alex, J. Doerschel, R. Schlage, J. Cryst. Growth 137, 278 (1994).

[3] M. Berkowski, J. Fink-Finowicki, M. Gutowski, in: Proc. III KK KOWBAN'96 (Third Domestic Conference on Computer Aided Scientific Research), Polanica Zdrój (Poland) 1996, Wrocławskie Towarzystwo Naukowe, Wrocław 1996, p. 115. 